\title{
COVID-19 pandemic and biological therapy in rheumatologic disorders: how to deal with?
}

\author{
Z. Ahmadinejad ${ }^{1,2}$, R. Assari ${ }^{3}$, N. Ayoobi Yazdi2,4, S.-H. Mazloomi², \\ P. Javanshayani ${ }^{2}$, H. Khalili Afousi ${ }^{5}$, V. Ziaee ${ }^{3,6}$ \\ ${ }^{1}$ Department of Infectious Disease, Tehran University of Medical Science, Tehran, Iran; \\ 2Imam Khomeini Complex Hospital, Tehran, Iran; ${ }^{3}$ Rheumatology Research Center, Tehran University of \\ Medical Science, Tehran, Iran; ${ }^{4}$ Department of Radiology, Tehran University of Medical Science, Tehran, Iran; \\ ${ }^{5}$ Department of Clinical Pharmacy, Tehran University of Medical Science, Tehran, Iran; \\ ${ }^{6}$ Department of Pediatrics, Tehran University of Medical Science, Tehran, Iran
}

\begin{abstract}
SUMMARY
The outbreak of coronavirus disease 2019 (COVID-19) has involved more than 159 countries and more than 5 million people worldwide. A 40-year-old man with a history of rheumatoid arthritis treated with prednisolone, Disease-Modifying Anti-Rheumatic Drugs (DMARDs), and biologic agents was admitted with chief complaints of fever, chills, malaise, myalgia, and dyspnea. Chest computed tomography showed bilateral subsegmental atelectasis and diffuse ground-glass opacities in both lungs inducing the suspicion of COVID-19 infection. The oro-nasopharynx swab sample for COVID-19 polymerase chain reaction was positive. In addition to supportive care, lopinavir/ritonavir 400/100 mg twice daily and oseltamivir (75 mg) twice daily were started in combination with a starting dose of hydroxychloroquine $(400 \mathrm{mg})$. The methotrexate dose was decreased, and the dose of prednisolone was increased to $30 \mathrm{mg}$ for 10 days. Azathioprine and adalimumab were continued at previous doses. The use of biologic agents and DMARDs in rheumatic patients is a serious challenge in the COVID-19 pandemic. In conclusion, during the COVID-19 pandemic, due to the key roles of cytokines in the promotion of the disease, the rheumatic patients may benefit from continuing their previous treatment, which may have protective effects.
\end{abstract}

Key words: COVID-19 pandemic; biological therapy; rheumatologic disorders.

\section{INTRODUCTION}

The coronavirus disease 2019 (COV-

ID-19) was declared to be a global pandemic on March $11^{\text {th }}, 2020$ due to its rapid global spread (1). Iran reported the first death officially on February $19^{\text {th }}, 2020$ (2). As of April $8^{\text {th }}, 2020,62,589$ confirmed cases and 3,872 deaths due to the severe acute respiratory syndrome coronavirus 2 (SARS-CoV-2) were reported in Iran (3).

One of the serious complications of COVID-19 is SARS with $10 \%$ mortality (4). The severity of the disease depends on the overexpression of the inflammatory responses rather than the pathogenicity of the virus (5).

The CD4 and CD8 T cells are reduced in the peripheral blood in COVID-19 pa- tients. Nevertheless, increased tissue CD4 $\mathrm{T}$ cells with a high concentration of proinflammatory capacities (Th17) and CD8 T cells with a high concentration of cytotoxic granules can cause organ damage (6).

Both connective tissue disorders and the recommended immunosuppressive therapy attenuate the responses of the immune system. Rheumatologists are currently facing the big challenge of the management of patients with rheumatologic disorders affected by COVID-19.

\section{CASE REPORT}

A 40-year-old man with a history of rheumatoid arthritis (RA) was referred to our hospital with chief complaints of fever, chills, malaise, myalgia, and dyspnea for
Corresponding author: Vahid Ziaee

Division of Pediatric Rheumatology, Children's Medical Center, No. 62 Dr. Gharib St., Keshavarz Blvd, Tehran 14194, IR Iran

E-mail: ziaee@tums.ac.ir ; ziaeev@gmail.com 
3-4 days, non-bloody diarrhea and transient dysuria without cough, nausea, and vomiting. He was a clerk in the West Azerbaijan province, Iran, of Azeri ethnicity. His medical history showed that his RA started when he was 26 years old, and there was no previous history of lung disease. He was a non-smoker. During the physical examinations, the patient was conscious, with no tachypnea (RR: $20 \mathrm{~min}$ ). Some vesicular skin lesions in the left groin and the middle line of the lumbar region were detected. His body temperature was $38.5^{\circ} \mathrm{C}$, blood pressure was $110 / 90 \mathrm{mmHg}, \mathrm{SpO}_{2}$ was $85 \%$, and pulse rate was 110 beats/min.

He had a history of chronic, severe RA for 14 years and was under treatment with adalimumab $40 \mathrm{mg}$ biweekly, prednisolone $15 \mathrm{mg}$ daily, methotrexate $15 \mathrm{mg}$ weekly, hydroxychloroquine $200 \mathrm{mg}$ daily, azathioprine $50 \mathrm{mg}$ daily, and folic acid $1 \mathrm{mg}$ daily, acetazolamide (because of high intraocular pressure due to prolonged usage of glucocorticoids) $250 \mathrm{mg}$ daily, and metoprolol (because of the history of tachycardia and hypertension), $50 \mathrm{mg}$ daily.

After admission on February, $29^{\text {th }}$ with suspicion of COVID-19, an imaging investigation was planned. Although he had normal chest X-rays, bilateral subsegmental atelectasis, and diffuse ground-glass opacities in both lungs (the main feature of COVID-19 pneumonia) (7) were reported in chest computed tomography (CT). No pleural and parenchymal effusion was seen (Figure 1). Oro/nasopharynx swab sample for COVID-19 polymerase chain reaction was positive. Over the first two days of admission, oxygen was delivered by nasal cannula and gradually tapered until the third day, when the patient was weaned off the respirator.

Supportive care was associated with lopinavir/ritonavir $(400 / 100 \mathrm{mg})$ twice daily, and oseltamivir $(75 \mathrm{mg}$ ) twice daily (according to the national guideline) (8), which were started in combination with only the starting dose of hydroxychloroquine (400 mg). However, hydroxychloroquine was stopped due to a probable interaction with the other drugs, such as lopinavir/ritonavir and QT prolongation.
Skin eruptions were compatible with herpes zoster infection. Intravenous acyclovir $(750 \mathrm{mg}$ ) every $8 \mathrm{~h}$ for $72 \mathrm{~h}$ and then valacyclovir $(1000 \mathrm{mg})$ three times a day for 10 days were administered. The methotrexate dose was decreased to $10 \mathrm{mg} / \mathrm{week}$, while prednisolone was increased to $30 \mathrm{mg} /$ day for 10 days for the prevention of possible RA flare-ups. Azathioprine and adalimumab were continued at previous doses.

Laboratory findings were as follows: WBC $=10.8 \times 10^{9} / \mathrm{L}$ (lymphocytes $16 \%$ ), hemoglobin $(13.4 \mathrm{~g} / \mathrm{L})$, platelet count $=163 \times 10^{9} / \mathrm{L}$,

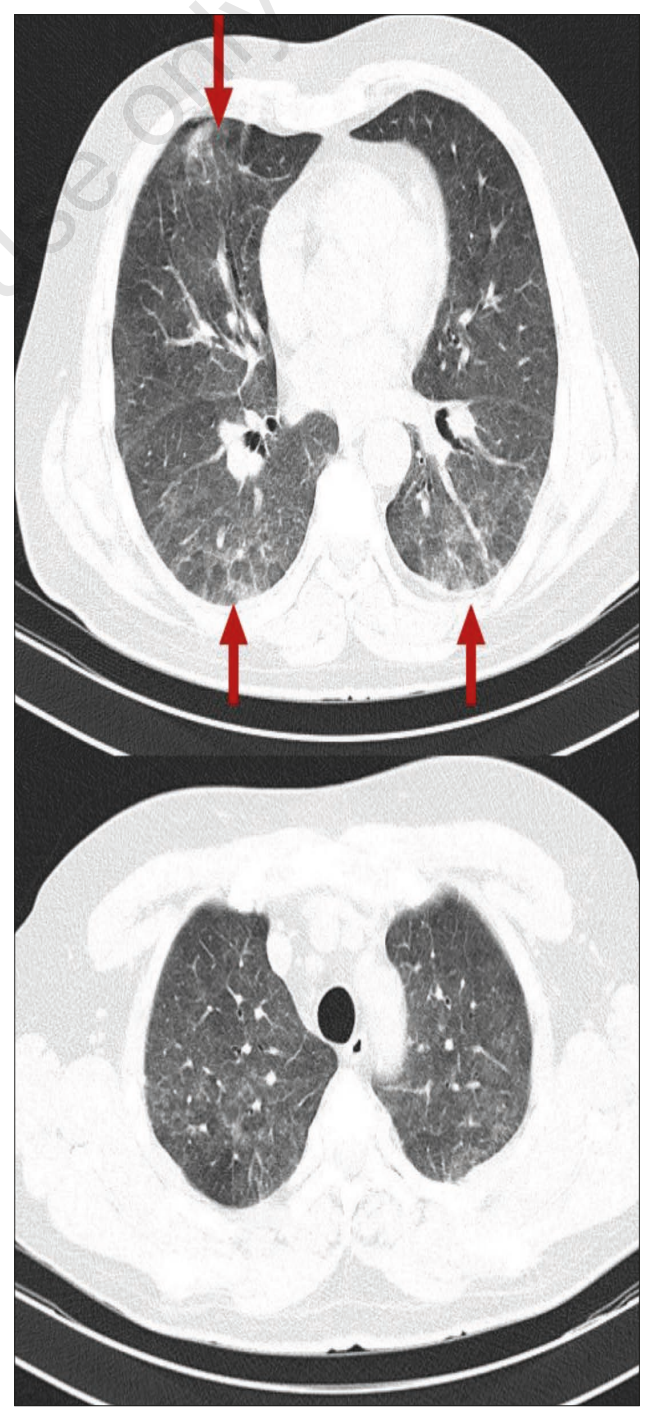

Figure 1 - Chest computed tomography image of our. Bilateral subsegmental atelectasis at the basis of the lungs (arrows) and diffuse ground glass opacities in both lungs were observed. 
erythrocyte sedimentation rate $(\mathrm{ESR})=10$ $\mathrm{mm} / \mathrm{h}$, C-reactive protein $(\mathrm{CRP})=97 \mathrm{mg} / \mathrm{L}$. Other laboratory findings, including blood culture, serum electrolytes, and renal and liver function tests were within the normal range. Evaluation of Mycobacterium tuberculosis infection was performed yearly, considering the patient was receiving antitumor necrosis factor (TNF), glucocorticoids, and immunosuppressive agents, and resulted always negative.

The patient was discharged from our hospital 4 days later in good conditions. $\mathrm{He}$ was advised to take lopinavir/ritonavir and oseltamivir for 10 and 5 days, respectively. Medications for his underlying disease (RA) were continued during his hospitalization and after discharge at previous doses. After 3 weeks, he was stable and symptom-free.

\section{DISCUSSION AND CONCLUSIONS}

The coronavirus penetrates into the cells through the angiotensin-converting enzyme 2 (ACE2) receptor and causes tissue damage. The loss of ACE2 function activates TNF $\alpha$ enzyme activators, and subsequently increases the expression of adhesion molecules and initiates the proinflammatory pathway (9). Both SARS-CoV and SARS-CoV-2 have the same host cell receptor (ACE2), and a similar pathway with a higher affinity for SARS-CoV-2 (10). The effectiveness of S protein of SARS-CoV on the amount of TNF $\alpha$ cytokine production depends on the quantity of ACE2 receptors on the cell surfaces. Some organs, such as lungs, kidneys, and the small intestine have a higher level of ACE2 receptors and develop more significant manifestations (10). Cytokines release syndrome (CRS) with an increased activity of $\mathrm{T}$ cells occurs in malignancies, transplantations, rheumatologic disorders, infections caused by influenza virus and coronavirus with a broad spectrum of manifestations from mild flu-like symptoms to severe systemic inflammation, which may be associated with decreased blood pressure, disseminated intravascular coagulation (DIC), and multiple organ failure. Lungs are one of the most important organs, and pulmonary involvement can range from mild manifestations, such as fever, cough, and tachypnea, to severe presentations, such as SARS (11).

In infection, cell lysis and release of cytokines, such as TNF, IL6, and INFy, activate the innate immune system to produce more and more cytokines, which mechanism may even progress to macrophage activation syndrome (MAS), as a cytokines storm. The key cytokine in this process is IL6, which causes cardiomyopathy, vascular leakage, DIC, and other severe complications. Therefore, anti-IL6 and glucocorticoids (GC) are effective treatments. However, the suppression of the immune system (specially the innate system) that facilitate serious infections should be done cautiously $(8,12,13)$.

Clinical studies to assess the role of the anti-IL6 agent tocilizumab in the management of COVID-19 are currently underway (12). In addition, the role of INF suppression, as a promoter cytokine in the cytokine storm, was investigated (14). Cyclosporine is likely to suppress virus replication (15) and is one of the main treatments in MAS and cytokines storm, since it can inhibit the early phase of $\mathrm{T}$ cell activation and proliferation, and IL2 production $(16,17)$. Glucocorticoids are another important treatment in MAS. They (glutamine synthetase) may increase the risk of contracting COVID-19 in rheumatic patients but should not be stopped $(18,19)$. However, there is some evidence that GC are useful in cytokine release syndrome, septic shock, and myocarditis in COVID $(16,17,20,21)$. Thus, the Endocrine Society recommended to double GC dose also for the prevention of secondary adrenal insufficiency (22). In our patient, because of varicella-zoster virus infection, methotrexate dose was decreased and GC dose doubled, also for the prevention of possible RA flare-ups $(23,24)$.

Nowadays, hydroxychloroquine (HCQ) is used as one of the main medications in the treatment of COVID-19 that may suppress virus replication. Indeed, it changes the $\mathrm{pH}$ of antigen-presenting cells, decreases 
the activation of dendritic cells and of the inflammatory process by blocking the tolllike receptors (25). In our patient, the dose of HCQ was doubled only in the first day and with caution, because its interaction with lopinavir/ritonavir may result in QT interval prolongation.

TNF $\alpha$ causes fever, myalgia, diarrhea, cardiomyopathy, and SARS in CRS. TNFo, as an initiator of the cytokine cascade in the pulmonary tissue, causes more cytokine production by stimulation of the innate immune system, and consequently, tissue damage. The anti-TNF $\alpha$ agents inhibit the promotion of pulmonary manifestations, such as ARDS, in mice (26-30). Our patient was treated with anti-TNF $\alpha$ (adalimumab) biweekly. Although he was infected with SARS-CoV-2, the biologic agent doses and its frequency of administration were not changed.

On the other hand, in patients with rheumatic diseases under treatment with antiTNF $\alpha$ agents, the suspension of biologics may result in the flare-up of the background connective tissue disease. Nevertheless, the discontinuation or continuation of biologics and non-biologic medicines in patients with rheumatic diseases and COVID-19 infection depends on patient's characteristics and comorbidities. In conclusion, in the COVID-19 pandemic, because of the key roles of cytokines in the promotion of the disease, the rheumatic patients may benefit from continuing the previous treatment, which may exert protective effects.

\section{Conflict of interest}

The authors declare no conflict of interest.

\section{Ethical approval}

This case report was approved by TUMS research ethical committee (Approval ID: IR.TUMS.VCR.REC.1399.126). The patient gave the written informed consent to report his data.

\section{REFERENCES}

1. WHO Director. General's opening remarks at the media briefing on COVID-19; 11 March 2020. Available from: https://www.who.int/ dg/speeches/detail/who-director-general-sopening-remarks-at-the-media-briefing-oncovid-19, 11-march-2020

2. The Provincial Committee on COVID-19 Epidemiology in Shahrekord University of Medical Sciences. Daily situation report on coronavirus disease (COVID-19) in Iran. N. 18; April 6, 2020. Available from: http://corona.behdasht. gov.ir/files/site1/files/Factsheet18-En.pdf

3. WordlOMeter. COVID-19 Coronavirus pandemic. Available from: https://www.worldometers.info/coronavirus/

4. Kaiazek TG, Erdman D, Goldsmith CS, et al. A novel coronavirus associated with severe acute respiratory syndrome. $\mathrm{N}$ Engl $\mathrm{J}$ Med. 2003; 15: 1953-60.

5. Huang C, Wang Y, Li X, et al. Clinical features of patients infected with 2019 novel coronavirus in Wuhan, China. Lancet. 2020; 395: 497506.

6. Zhe Xu, Lei Shi, Yijin Wang, et al. Pathological findings of COVID-19 associated with acute respiratory distress syndrome. Lancet Respir Med. 2020 Feb 18 [Epub ahead of print].

7. Hani C, Trieu NH, Saab I, et al. COVID-19 pneumonia: A review of typical CT findings and differential diagnosis. Diagn Intervent Imaging. 2020; 101: 263-8.

8. Health and Treatment Deputy of the Ministry of Health and Medical Education (2020). Guideline for the diagnosis and treatment of COVID-19 in outpatients and inpatients. Available from: http://dme.behdasht.gov.ir/ uploads/Felo_Tashkish.pdf

9. Zhu L, Carretero OA, Xu J, et al. Activation of angiotensin II type 2 receptor suppresses TNF- $\alpha$-induced ICAM- 1 via NF-B: possible role of ACE2. Am J Physiol Heart Circ Physiol. 2015; 309: 827-34.

10. Wang P-H, Cheng Y. Increasing host cellular receptor angiotensin converting enzyme2 (ACE2) expression by coronavirus may facilitate 2019-nCov infection. bioRxiv 2020.02.24. 963348; doi: https://doi.org/10.1101/2020.02. 24.963348

11. Shimabukuro-Vornhagen A, Gödel P, Subklewe M, et al. Cytokine release syndrome. J Immunother Cancer. 2018; 6: 56.

12. Anaka T, Narazaki M, Kishimoto T. Immunotherapeutic implications of IL-6 blockade for cytokine storm. Immunotherapy. 2016; 8: 959-70.

13. Chinese Clinical Trial Registry. A multicenter, randomized controlled trial for the efficacy and safety of tocilizumab in the treatment of new coronavirus pneumonia (COVID-19). Available from: http://www. chictr.org.cn/showprojen. aspx ?proj=49409 Accessed: March 5, 2020.

14. Stebbing J, Phelan A, Griffin I, et al. COVID-19: combining antiviral and anti-inflam- 
matory treatments. Lancet Infect Dis. 2020 Feb 27 [Epub ahead of print].

15. Adriaan H, de Wilde, Jessika C, et al. Cyclosporin A inhibits the replication of diverse coronavirus. J Gen Virol. 2011; 92: 2542-8.

16. Boom V, Anton J, Lahdenne P, et al. Evidence-based diagnosis and treatment of macrophage activation syndrome in systemic juvenile idiopathic arthritis. Ped Rheumatol. 2015; 13: 55.

17. Assari R, Sadeghi P, Mirmohammadsadeghi A, et al. Macrophage activation syndrome as a complication of rheumatologic disorders, a report from Iran. Reumatismo. 2019; 71: 189-98.

18. Centers for Disease Control and Prevention. Interim clinical guidance for management of patients with confirmed 2019 novel coronavirus (2019-nCoV) infection; Updated February $12,2020$.

19. Alhazzani W, Møller MH, Arabi YM, et al. Surviving Sepsis Campaign: guideline on the management of critically ill adult with Coronavirus Disease 2019 (COVID-19). Intensive Care Med. 2020; 46: 854-87.

20. Centers for Disease Control and Prevention (CDC). Interim Clinical Guidance for Management of Patients with Confirmed Coronavirus Disease (COVID-19). Available from: https:// www.cdc.gov/coronavirus/2019-ncov/hcp/clinical-guidance-management-patients.html

21. ClinicalTrials.gov. Efficacy and safety of corticosteroids in COVID-19. Available from: https://clinicaltrials.gov/ct2/show/NCT04273321

22. Endocrine Society. Individuals taking class of steroid medications at high risk for $\mathrm{COV}$ ID-19; March 31, 2020. Available from: https://www.endocrine.org/news-and-advo- cacy/news-room/2020/individuals-takingclass-of-steroid-medications-at-high-riskfor-covid

23. Colson P, Rolain JM, Lagier JC, et al. Chloroquine and hydroxychloroquine as available weapons to fight COVID-19. Int J Antimicrob Agents. 2020 Mar 4: 105932 [Epub ahead of print].

24. Favallia EG, Ingegnolia F, Lucia OD, et al. COVID-19 infection and rheumatoid arthritis: Faraway, so close! Autoimmun Rev. 2020 [Epub ahead of print].

25. Robinson PC, Yazdany J. The COVID-19 global rheumatology alliance: collecting data in a pandemic. Nature Rev Rheumatol. 2020; 16: 293-4.

26. Peiris JSM, Chu CM, Cheng VCC, et al. Clinical progression and viral load in a community outbreak of coronavirus-associated SARS pneumonia: a prospective study. Lancet. 2003; 361: 1767-72.

27. Nichols JM, Poon LLM, Lee KC, et al. Lung pathology of fatal severe acute respiratory syndrome. Lancet. 2003; 361: 1773-8.

28. Hussell T, Pennycook A, Openshaw PJ. Inhibition of tumor necrosis factor reduces the severity of virus-specific lung immunopathology. Eur J Immunol. 2001; 31: 2566-73.

29. Yanik G, Hellerstedt B, Custer J, et al. Etanercept (Enbrel) administration for idiopathic pneumonia syndrome after allogeneic hematopoietic stem cell transplantation. Biol Blood Marrow Transplant. 2002; 8: 39.

30. Iranian Registry of Clinical Trials. Evaluation the efficacy of tumor necrosis factor alpha inhibitor in COVID-19 outcome: a prospective clinical trial study. Available from: https://irct. ir/trial/46551. 